\title{
Long-Term (10-Year) Gastrointestinal and Genitourinary Toxicity after Treatment with External Beam Radiotherapy, Radical Prostatectomy, or Brachytherapy for Prostate Cancer
}

\author{
Grant K. Hunter, ${ }^{1}$ Chandana A. Reddy, ${ }^{1}$ Eric A. Klein, ${ }^{2}$ Patrick Kupelian, ${ }^{3}$ \\ Kenneth Angermeier, ${ }^{2}$ James Ulchaker, ${ }^{2}$ Nabil Chehade, ${ }^{4}$ Andrew Altman, ${ }^{4}$ and Jay P. Ciezki ${ }^{1}$ \\ ${ }^{1}$ Cleveland Clinic Department of Radiation Oncology, 9500 Euclid Avenue, Cleveland, OH 44195, USA \\ ${ }^{2}$ Cleveland Clinic GlickmanUrological and Kidney Institute, 9500 Euclid Avenue, Cleveland, OH 44195, USA \\ ${ }^{3}$ UCLA Jonsson Comprehensive Cancer Center, 200 UCLA Medical Plaza, Suite B265, Los Angeles, CA 90095-6951, USA \\ ${ }^{4}$ Department of Urology, Kaiser Permanente, Ohio, 12301 Snow Road, Parma, OH 44130, USA
}

Correspondence should be addressed to Jay P. Ciezki, ciezkij@ccf.org

Received 23 November 2011; Revised 29 January 2012; Accepted 30 January 2012

Academic Editor: James L. Gulley

Copyright ( 2012 Grant K. Hunter et al. This is an open access article distributed under the Creative Commons Attribution License, which permits unrestricted use, distribution, and reproduction in any medium, provided the original work is properly cited.

\begin{abstract}
Objective.To examine gastrointestinal (GI) and genitourinary (GU) toxicity profiles of patients treated in 1999 with external beam radiotherapy (RT), prostate interstitial brachytherapy (PI) or radical prostatectomy (RP). Methods. TThe records of 525 patients treated in 1999 were reviewed to evaluate toxicity. Late GI and GU morbidities were graded according to the RTOG late morbidity criteria. Other factors examined were patient age, BMI, smoking history, and medical co-morbidities. Due to the low event rate for late GU and GI toxicities, a competing risk regression (CRR) analysis was done with death as the competing event. Results. Median follow-up time was 8.5 years. On CRR univariate analysis, only the presence of DM was significantly associated with GU toxicity grade $>2(P=0.43, \mathrm{HR} 2.35,95 \% \mathrm{Cl}=1.03-5.39)$. On univariate analysis, $\mathrm{RT}$ and $\mathrm{DM}$ were significantly associated with late GI toxicity. On multivariable analysis, both variables remained significant (RT: $P=0.038, \mathrm{HR}=4.71, \mathrm{CI}=1.09-20.3$; DM: $P=0.008, \mathrm{HR}=3.81,95 \% \mathrm{Cl}=1.42-10.2)$. Conclusions. Late effects occur with all treatment modalities. The presence of DM at the time of treatment was significantly associated with worse late GI and GU toxicity. RT was significantly associated with worse late GI toxicity compared to PI and RP.
\end{abstract}

\section{Introduction}

For patients with localized prostate cancer, treatment options include active surveillance, radical prostatectomy (RP), external beam radiation therapy (RT), or low-doserate prostate interstitial brachytherapy (PI). There is data showing that in low-risk disease, excellent prostate-specific antigen (PSA) relapse-free survival outcomes are obtained with a low risk of significant treatment-related morbidity [1-4]. Low-risk patients do well with any of the three treatment modalities but there is no data comparing late toxicity. Due to lack of randomized data comparing these modalities, treatment decisions are often made by patient or physician preference based on the side effect profiles exhibited by each treatment type. While all modalities have generally similar toxicity profiles, there are slight differences between each that can impact long-term quality of life [5]. There are reports comparing the late toxicity rates between different types of radiation treatment options with noted improvement in morbidity with newer treatment techniques such as intensity-modulated radiation therapy (IMRT) [6]. However, there have been few reports comparing potential long-term toxicity profiles of patients treated with radiation treatment modalities to those who have undergone surgical 
resection. In the present paper we compare our data on toxicity for patients treated in 1999 with RP, RT (IMRT), or PI.

\section{Materials and Methods}

The records of 525 patients treated in 1999 were reviewed to evaluate toxicity profiles. The year 1999 was chosen to allow for sufficient potential follow-up time, and by that time, all the three treatment programs (RP, RT, and PI) had been well established for years. Of all patients treated during the 1999 calendar year we excluded 42 patients for whom we had no follow-up data, with the excluded patients being evenly distributed among the three methods of treatment. All patients were identified from our prospectively maintained, IRB-approved prostate cancer registry. Of the 483 patients with clinical followup, 116 (24\%) were treated with PI, 195 (40\%) with RP, and 172 (36\%) with RT.

Each patient's medical record was examined with attention to visits to his urologist, radiation oncologist, internal medicine, or family care physician and any gastroenterologist since the time of treatment. Late toxicity was defined as toxicity occurring at least six months after treatment. Gastrointestinal (GI) and genitourinary (GU) morbidity profiles were specifically examined and graded according to the RTOG acute and late morbidity scoring criteria. The RTOG morbidity scoring criteria is a numeric scoring system with scores ranging from grade 1 (mild morbidity not requiring any intervention) to grade 5 (death), with specific criteria for GI and GU side effect profiles. These criteria were not effective in describing toxicities experienced by the group of patients treated with surgical resection, so the National Cancer Institute's Common Terminology Criteria for Adverse Events, (CTCAE) version 4.0, was used to grade surgical patients' late morbidity. The CTCAE is comparable, but not identical to the RTOG late toxicity scoring system. The CTCAE is generally more descriptive and comprehensive compared to the RTOG late morbidity scoring criteria; however there is significant overlap in the described grading systems. For example, the RTOG criteria donot mention a surgical intervention in their late GI scoring criteria save in the case of grade 3. However, the RTOG grade 2 late GI scoring criteria include: "moderate diarrhea and colic; bowel movement $>5$ times daily; excessive rectal mucus or intermittent bleeding." When patients presented for followup with intermittent bleeding after RT we felt this was clearly a grade 2 toxicity and not a grade 3, which the RTOG criteria list as being: "obstruction or bleeding requiring surgery." This description of late GI toxicity that includes intermittent bleeding is similar to the CTCAE grade 2 GI toxicity which is listed under rectal hemorrhage as: "moderate symptoms; medical intervention or minor cauterization indicated." The late GU toxicity criteria are also slightly different as the RTOG criteria never mention surgical intervention at all, and late grade 2 toxicity incorporates: "moderate frequency; generalized telangiectasia; intermittent macroscopic hematuria." The CTCAE grade 2 hematuria is listed as including "symptomatic, urinary catheter or bladder irrigation indicated; limiting instrumental ADL." Again, the scoring systems are comparable, but not identical.

Upon review of the patient's medical record, at any visit wherein toxicity was addressed or brought up by the patient, the specific GI or GU toxicity was graded according to the above criteria. Other factors examined were patient age, body mass index (BMI), smoking history, and medical comorbidities including presence of diabetes mellitus (DM), peripheral vascular disease, and connective tissue disease.

Due to the low event rate and the potential for patients to have died before developing a late GU or GI toxicity, a competing risk regression (CRR) analysis was done for each toxicity endpoint with death as the competing event [7]. The final multivariable model was built using the forward, stepwise procedure. Factors with a $P$ value $<0.05$ on a univariate analysis were included in a multivariable analysis. Variables having a $P$ value of $<0.05$ remained in the final model. Cumulative incidence rates of late GU and GI were calculated, and comparisons among the three treatment groups were made using Gray's test [8]. The competing risk regression analysis and the cumulative incidence rate comparisons were performed using $\mathrm{R}$ version 2.8.1 (The $\mathrm{R}$ Foundation for Statistical Computing, Vienna, Austria). All other statistical analyses were done using SAS version 9.2 (SAS Institute, Cary, NC, USA).

2.1. Radical Prostatectomy Group. Patients in the surgical group underwent RP under the care of surgeons in the department of urology. The majority underwent open, retropubic radical prostatectomies. Twelve $(6.2 \%)$ patients underwent a laparoscopic prostatectomy or robotic-assisted prostatectomy. Ninety-eight $(50.3 \%)$ patients underwent a bilateral nerve sparing prostatectomy, $20(10.3 \%)$ had unilateral nerve sparing, and $77(39.5 \%)$ did not undergo a nerve-sparing prostatectomy. Twenty-three patients (11.8\%) received adjuvant salvage radiation therapy to the prostate.

2.2. External Beam Radiation Therapy Group. All but 11 (6.4\%) patients in the EBRT group were treated with IMRT. The patients were treated under the care of two radiation oncologists with the general treatment planning guidelines previously described $[9,10]$. In brief, the patients were treated with a five-field IMRT plan to a total dose of $70 \mathrm{~Gy}$ via 28 daily fractions of $2.5 \mathrm{~Gy}$. This dose and hypofractionated regimen was the standard at the Cleveland Clinic in 1999. Hypofractionated IMRT is one method of delivering external beam radiotherapy. It remains under investigation, with a phase III trial (RTOG 0415) conducted by the RTOG, with long-term data maturing. The 11 patients who were not treated with IMRT were treated with a conformal radiation treatment plan to a total dose of 78 Gy via 39 daily fractions of $2.0 \mathrm{~Gy}$.

The treating physician delineated all target and normal tissue volumes, with low-risk patients having the target defined as the prostate only and high-risk patients having the prostate and seminal vesicles included as the target. The expansions to obtain a planning target volume (PTV) from 
Table 1: Patient Characteristics.

\begin{tabular}{|c|c|c|c|c|}
\hline Characteristic & $\mathrm{RP}(n=195)$ & $\mathrm{RT}(n=172)$ & $\mathrm{PI}(n=116)$ & $P$ value \\
\hline Median age (yrs), (range) & $62,(42-75)$ & $68,(48-84)$ & $69,(47-78)$ & $<0.0001$ \\
\hline Median follow-up time (yrs), (range) & $8.6,(1.7-11.2)$ & $8.9,(0.13-11.4)$ & $8.1,(0.16-11.5)$ & 0.08 \\
\hline Median body mass index $\left(\mathrm{m} / \mathrm{kg}^{2}\right)$, (range) & 26.92, (19-39) & $27.57,(18-42)$ & $27.2,(20-42)$ & 0.07 \\
\hline Ever smoked (\%) & 65.1 & 52.9 & 63.8 & 0.10 \\
\hline Diabetic (\%) & 6.7 & 15.1 & 10.3 & 0.02 \\
\hline Peripheral vascular disease (\%) & 1.5 & 3.5 & 7.8 & 0.02 \\
\hline Connective tissue disease (\%) & 0.5 & 0 & 0 & 0.48 \\
\hline Mean charlson score (range) & $0.3,(0-3)$ & $0.5,(0-7)$ & $0.8,(0-7)$ & $<0.0001$ \\
\hline Androgen deprivation (\%) & 16.9 & 63.4 & 30.2 & $<0.0001$ \\
\hline Risk category $(\%)$ & & & & $<0.0001$ \\
\hline Low & 50.8 & 30.8 & 75 & \\
\hline Intermediate & 25.1 & 29.7 & 25 & \\
\hline High & 24.1 & 39.5 & 0 & \\
\hline
\end{tabular}

the prostate or prostate + seminal vesicles were $4 \mathrm{~mm}$ posteriorly, $8 \mathrm{~mm}$ laterally, and $5 \mathrm{~mm}$ in all other dimensions. The dose constraints used for treatment planning included the target volume (PTV) to $70 \mathrm{~Gy}$ with a range of 65 to $78 \mathrm{~Gy}$ with the dose prescribed to an isodose line covering the target ranging from $82.0 \%$ to $90.0 \%$. The limits that were used for bladder were no more than $30 \%$ to receive greater than 55 Gy with maximum level at 74 Gy and no more than $30 \%$ of rectum to be greater than $50 \mathrm{~Gy}$ with maximum dose of 74 Gy.

For daily localization, after set-up via triangulation marks, all patients underwent ultrasound image guidance using the BAT transabdominal ultrasound system. The system consists of a B-mode transabdominal ultrasound probe attached to a precision tracking arm with greater details described previously [9].

2.3. Prostate Brachytherapy Group. Patients in the brachytherapy group underwent PI under the care of one of two radiation oncologists. The brachytherapy procedure has been described previously [11]. In brief, patients were treated with a dose of $144 \mathrm{~Gy}$ prescribed to the prostate using I-125 monotherapy. All patients underwent intraoperative planning using a transrectal ultrasound probe for creation of an optimal seed loading pattern that would allow for delivery of the prescription dose and conform to established dose constraints for the surrounding normal tissues. The target volume was defined on the intraoperative ultrasound axial images and consisted of the prostate only for low-risk patients and the prostate and $0.5-1 \mathrm{~cm}$ of the proximal seminal vesicles for intermediate- and highrisk patients. A postprocedure CT scan was performed one month following implantation for the purpose of dosimetric evaluation. Dosimetric guidelines followed for evaluation of the brachytherapy consisted of minimum dose to $90 \%$ of the prostate PTV (D90) between $90 \%$ and $130 \%$ of the prescribed dose, at least $80 \%$ of the prostate PTV to get at least $100 \%$ of the prescribed dose, and the urethral dose should be limited to $150 \%$ of the target dose. The dosimetric constraint for the rectum was defined as the volume of rectum receiving $100 \%$ of the prescribed dose and it was limited to be less than $1 \mathrm{cc}$.

Follow-up evaluations were done one month after treatment for all three modalities. Subsequent follow-up examinations were done between three to six months and then yearly for five years. Following five years, followup with either the treating urologist or radiation oncologist was sporadic; however followup with the primary care physician was reviewed and coded according to the abovementioned toxicity criteria. Most often, if any GI or GU events occurred and the patient was not currently following with his radiation oncologist or urologist, the patient was referred back for followup upon incidence of the toxicity. The median followup time for all groups of patients was 8.5 years (range 0.1-11.5 years). The median follow-up times for each group are as follows: RP: 8.6 years, PI: 8.1 years, EBRT: 8.9 years. The patient characteristics of all treatment groups are shown in Table 1.

\section{Results}

Overall, all three modalities were associated with relatively low late GI and GU toxicity rates. The frequency of late GU toxicity (grade 2 or higher) was $6.8 \%$ for all patients and was $4.3 \%$ for PI patients, $5.1 \%$ for RP patients, and $10.5 \%$ for RT patients. As demonstrated in Figure 1(a), the cumulative incidence of long-term GU toxicity (grade 2 or higher) was highest in patients treated with RT $(11.2 \%$ at 10 years). Patients treated with RP had the next highest cumulative incidence of GU toxicity, 5.5\% at 10 years, while the cumulative incidence at 10 years was $4.3 \%$ for PI. There were no GU toxicities noted beyond four years for PI patients. GU toxicities were observed more than eight years after treatment for RP patients. The varying types of late toxicities were slightly different following RP compared to those after PI or RT. The slight variations as well as the relative frequency are noted in Table 2.

In the competing risk regression univariate analysis, the presence of DM was associated with higher rates of GU toxicity $(P=0.043$, HR $2.35,95 \% \mathrm{CI}=1.03-5.39$, 
Cumulative incidence for late GU toxicity $(\geq 2)$ by treatment for patients treated in 1999

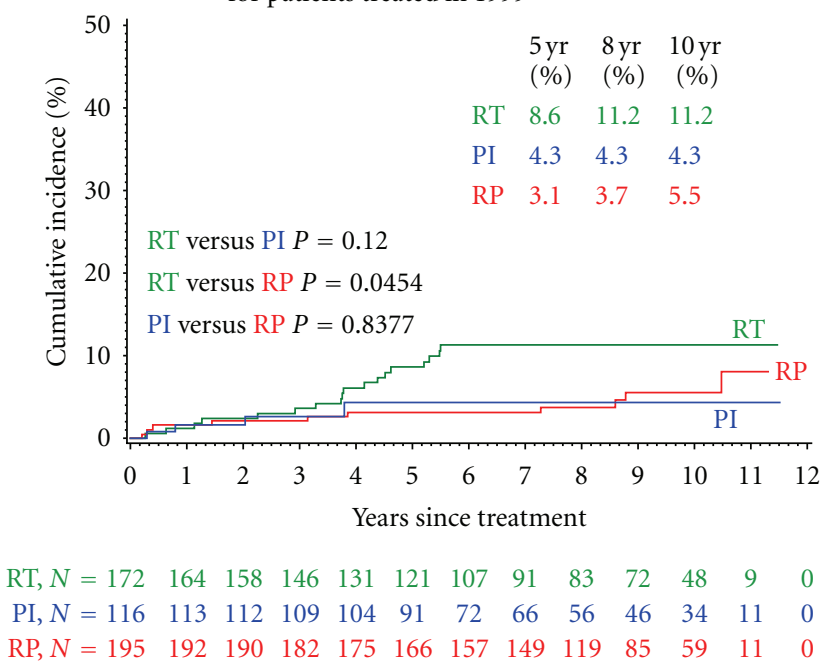

(a)
Cumulative incidence for late GI toxicity $(\geq 2)$ by treatment for patients treated in 1999

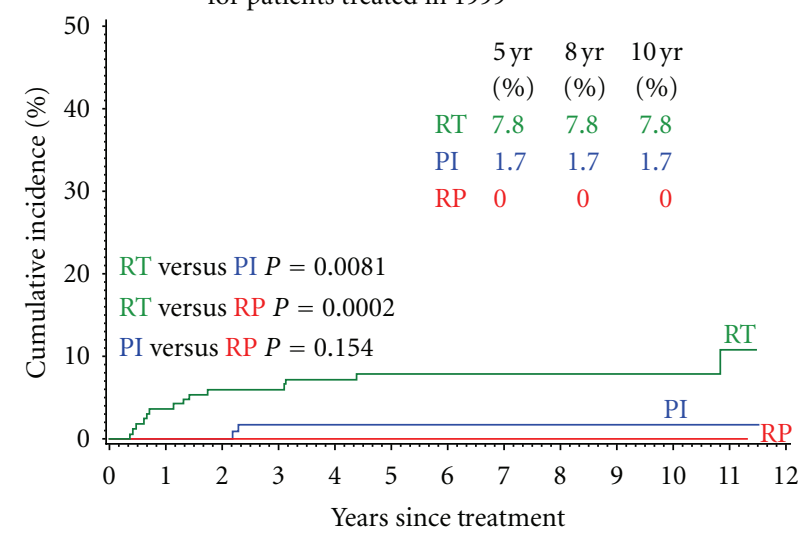

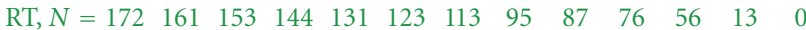

PI, $N=116 \quad 115 \quad 114110 \begin{array}{lllllllllll}106 & 92 & 73 & 67 & 57 & 46 & 34 & 10 & 0\end{array}$

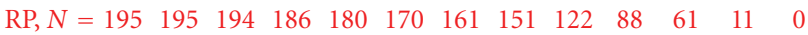

(b)

FIGURE 1: (a) Cumulative incidence curves of GU toxicity, $\geq$ grade 2 for patients treated in 1999 with external beam radiation therapy, radical prostatectomy, or prostate brachytherapy. (b) Cumulative incidence curves of GI toxicity, $\geq$ grade 2 for patients treated in 1999 with either external beam radiation therapy, radical prostatectomy, or prostate brachytherapy.

TABLE 2: Distribution of Toxicities Requiring Interventions (Corresponding to grade $\geq 2$ ).

\begin{tabular}{|c|c|c|c|}
\hline $\begin{array}{l}\text { Modality most common } \\
\text { toxicities }\end{array}$ & GU toxicities no. (\%) & $\begin{array}{l}\text { Modality most common } \\
\text { toxicities }\end{array}$ & GI toxicities no. (\%) \\
\hline \multicolumn{4}{|l|}{$\mathrm{RP}$} \\
\hline Urinary stricture & $6(3.0 \%)$ & & \\
\hline Incontinence & $2(1.0 \%)$ & & \\
\hline Urinary leakage & $2(1.0 \%)$ & & \\
\hline PI & & PI & \\
\hline Urinary stricture & $4(3.4 \%)$ & Rectal bleeding & $2(1.7 \%)$ \\
\hline Incontinence & $1(0.8 \%)$ & & \\
\hline Retention & $1(0.8 \%)$ & & \\
\hline RT & & RT & \\
\hline Frequency & $5(2.9 \%)$ & Rectal bleeding & $8(4.7 \%)$ \\
\hline Hematuria & $7(4.0 \%)$ & Radiation Proctitis & $4(2.3 \%)$ \\
\hline Retention & $6(3.5 \%)$ & Increased freq. & $2(1.1 \%)$ \\
\hline
\end{tabular}

Table 3(a)). DM was the only factor that was statistically significant with relation to the onset of late GU toxicity. Type of treatment was not significantly associated with late GU toxicity. The major causes of GU toxicity were increased frequency/irritation and varying degrees of incontinence. The vast majority of toxicities resolved with further followup or intervention.

There were no late GI toxicities (grade 2 or higher) observed for patients who underwent RP (Figure 1(b)). The frequency of late GI toxcitiy (grade 2 or higher) was 3.3\% for all other patients. For patients treated with PI the frequency was $1.7 \%$ and was $8.1 \%$ for patients treated with RT. The $10-$ year cumulative incidence rate of late GI toxicity was $7.8 \%$ in patients who had RT versus $1.7 \%$ for PI patients.

Since there were no events in the RP group, only the PI and RT patients were included in the CRR analysis for late GI toxicity grade $\geq 2$. On univariate analysis, RT and DM were significantly associated with late GI toxicity. On multivariable analysis, both variables remained significant (RT: $P=0.038, \mathrm{HR}=4.71,95 \% \mathrm{CI}=1.09-20.3$; DM: 
TABLE 3: (a) Competing risk regression for late GU toxicity $(\geq 2)$ for patients treated in 1999. (b) Competing risk regression for late GI toxicity $(\geq 2)$ for patients treated with RT or PI in $1999(n=282)$.

(a)

\begin{tabular}{lccc}
\hline Univariate analysis & $P$ value & $\begin{array}{c}\text { Hazard } \\
\text { ratio }\end{array}$ & $95 \%$ CI \\
\hline Treatment & & & \\
$\quad$ RP versus PI & 0.73 & 1.21 & $0.41-3.55$ \\
$\quad$ RT versus PI & 0.07 & 2.54 & $0.94-6.87$ \\
$\quad$ RT versus RP & 0.06 & 2.11 & $0.97-4.59$ \\
Age (continuous variable) & 0.12 & 1.04 & $0.99-1.10$ \\
Charlson score & 0.52 & 1.09 & $0.84-1.42$ \\
Peripheral vascular disease (yes & 0.42 & 1.81 & $0.43-7.62$ \\
versus no) & 0.043 & 2.35 & $1.03-5.39$ \\
$\begin{array}{l}\text { Diabetes (yes versus no) } \\
\text { Androgen deprivation (yes }\end{array}$ & 0.65 & 0.85 & $0.41-1.74$ \\
versus no) & 0.40 & 1.03 & $0.97-1.09$ \\
\hline Body mass index & & &
\end{tabular}

(b)

\begin{tabular}{lccc}
\hline Univariate analysis & $P$ value & $\begin{array}{c}\text { Hazard } \\
\text { ratio }\end{array}$ & $95 \%$ CI \\
\hline Treatment & & & \\
$\quad$ EBRT versus BT & 0.03 & 5.10 & $1.17-22.3$ \\
Age (continuous variable) & 0.27 & 1.05 & $0.96-1.14$ \\
Charlson score & 0.13 & 1.21 & $0.94-1.56$ \\
$\begin{array}{l}\text { Peripheral vascular disease (yes } \\
\text { versus no) }\end{array}$ & 0.85 & 1.22 & $0.17-9$ \\
$\begin{array}{l}\text { Diabetes (yes versus no) } \\
\text { Androgen deprivation (yes }\end{array}$ & 0.0051 & 4.2 & $1.54-11.5$ \\
$\begin{array}{l}\text { versus no) } \\
\text { Body mass index }\end{array} \quad 0.63$ & 0.78 & $0.29-2.11$ \\
$\quad$ Multivariable analysis & 0.88 & 0.99 & $0.83-1.17$ \\
$\begin{array}{l}\text { Diabetes (yes versus no) } \\
\text { EBRT versus BT }\end{array}$ & 0.008 & 3.81 & $1.42-10.2$ \\
& 0.038 & 4.71 & $1.09-20.3$ \\
\hline
\end{tabular}

$P=0.008, \mathrm{HR}=3.81,95 \% \mathrm{CI}=1.42-10.2$; Table $3(\mathrm{~b}))$. The major causes of GI toxicity were rectal bleeding and proctitis. The vast majority of toxicities resolved with further followup or intervention.

\section{Comment}

Our results show that patients treated with any of the three modalities had low rates of late toxicity. The median followup time of 103 months is longer than most retrospective series or cohort studies examining the toxicities associated with prostate cancer treatments. We appreciated an increase in both GU and GI long-term toxicity, in patients with preexisting DM. We also observed an increased incidence of long-term GI toxicity in patients treated with RT relative to patients treated with RP or PI that was significant on univariate and multivariable analysis. Our data confirmed that long-term GI toxicity is lower in RP patients compared with either modality of radiation treatment which correlates with data from multiple quality-of-life studies [12-14].

Diabetes has been shown to be a known risk factor for worse long-term toxicities after treatment for prostate cancer. In the CaPSURE study, a longitudinal disease registry of men with prostate cancer, men with DM who were treated with radical prostatectomy had worse urinary control over a follow-up period of two years [15]. Men with DM have been shown to have worse late GI and GU side effects after being treated with $3 \mathrm{D}$ conformal radiation therapy compared to nondiabetic men $[16,17]$. In a series from the British Columbia Cancer Agency, men treated with I-125 brachytherapy and who had DM were more likely to have late (median followup 57 months) symptomatic flares of GU toxicity as measured by the International Prostate Symptom Score (IPSS) [18].

This association of DM with worse long-term morbidity correlates with a report from Thong et al. [19]. They examined the data from the Prostate Cancer Outcomes Study (PCOS) with specific attention to the longitudinal effect of DM on the health-related quality of life (HRQOL) measures that were collected. With five years of followup, they reported that men with DM who were diagnosed prior to treatment had worse HRQOL scores across treatment groups of radical prostatectomy, radiation therapy, androgen deprivation, or watchful waiting. Furthermore, diabetic men had the lowest urinary control and sexual function scores over time. These differences in HRQOL remained constant over the five years evaluated. There are several reports that show the presence of DM leads to an increase in all-cause mortality among patients with prostate cancer, but not an increase in prostatecancer-related death, suggesting similar treatment efficacy [20-24]. Our data further adds that late toxicity is seen more often in patients with DM compared to those without DM.

Other quality-of-life data that compares all three treatment modalities most often lack prolonged followup. The PROST-QA trial had 24 months of followup and demonstrated the resolution of acute morbidity but did not describe further late toxicity [5]. In contrast with a study done by Miller et al. that was reported in 2005, our series of patients shows evolving long-term toxicity with all three types of treatment modalities [25]. Miller reported on patients who had received 3D-CRT, PI, or RP, with median time of followup ranging from 5.4 to 6.5 years since treatment and noted that patients who had undergone RP were unlikely to have further toxicity after a period of two years. Patients in this cohort who underwent RT or PI were more likely to have long-term emerging toxicity as reported by their patient-reported quality-of-life outcomes study. Our data, with median follow-up time ranging from 8.1 to 8.9 years since treatment, contradicts this finding as we found in our series that the cumulative incidence of GU toxicity for RP patients increased over time, even after five years. While this finding was novel, it is important to note that the cumulative rate was still low (5.5\% at 10 years), and this may be noted in the cohort reported by Miller with continued followup.

The relative increase in GI toxicity seen in patients who underwent RT compared to RP and PI could potentially be attributed to the hypofractionated dose of their IMRT. 
In 1999, when these treatments were performed, the institutional practice at the Cleveland Clinic was to treat prostate cancer via hypofractionated IMRT at $2.5 \mathrm{~Gy} /$ fraction. With the slightly larger dose per fraction compared to more traditional dosing (1.8-2.0 Gy/fraction), it could be hypothesized the late effects are results of the $2.5 \mathrm{~Gy} /$ fraction regimen as fraction size is the dominant factor in determining late effects from radiotherapy [26]. Earlier reports of the tolerability of the hypofractionated regimen show it to be comparable to more standard dose fractionation schemes with regard to disease control and toxicity rates $[27,28]$. These data are a continuation of followup of a part of the patient cohort described in the reports by Kupelian et al. and could simply show that with longer followup late GI toxicity will occur [28]. However, it is important to note that the cumulative incidence rate for this toxicity is still low. In a randomized trial published recently by Yeoh et al. patients treated with hypofractionated radiation ( $55 \mathrm{~Gy} / 20$ fractions) compared to conventionally fractionated radiation ( $64 \mathrm{~Gy} / 32$ fractions) had equivalent rates of GI and GU toxicity with prospective toxicity data accrued up to 60 months after treatment, suggesting further that hypofractionated radiation treatment has similar toxicity to conventional radiation therapy [29].

While this study lacks the thorough, regimental followup of prospective clinical trials, it does capture the sort of toxicity that will cause a patient to be referred for intervention (e.g., urinary strictures requiring dilation and rectal bleeding requiring cauterization). As such, this methodology will not adequately assess the subjective quality-of-life changes that equate to a grade I toxicity. It does, however, record the level of toxicity necessitating intervention and therefore gives a good estimate of the long-term cost in terms of objective quality-of-life changes as well as the cost in terms of medical resources used to deal with the sequelae of treatment. Further, since it is a retrospective study with physicianreported data there is a potential to underestimate the level of toxicity assessed. Patient-reported, prospective data would have been ideal but was not available. Our study is limited by the lack of follow-up data on sexual potency, which is an important factor in overall quality of life following treatment for prostate cancer.

\section{Conclusion}

Prostate cancer treatment is associated with low-level longterm GI and GU toxicity. While the incidence is small, the differences among modalities are significant. When assessing the effect of treatment, one must not only account for the cost of initial treatment, but also the cost of handling toxicity. The presence of diabetes adds to the risk of developing longterm toxicity.

\section{References}

[1] P. A. Kupelian, J. Ciezki, C. A. Reddy, E. A. Klein, and A. Mahadevan, "Effect of increasing radiation doses on local and distant failures in patients with localized prostate cancer," International Journal of Radiation Oncology Biology Physics, vol. 71, no. 1, pp. 16-22, 2008.
[2] A. Pollack, G. K. Zagars, G. Starkschall et al., "Prostate cancer radiation dose response: results of the M. D. Anderson phase III randomized trial," International Journal of Radiation Oncology Biology Physics, vol. 53, no. 5, pp. 1097-1105, 2002.

[3] M. J. Zelefsky, H. Chan, M. Hunt, Y. Yamada, A. M. Shippy, and H. Amols, "Long-term outcome of high dose intensity modulated radiation therapy for patients with clinically localized prostate cancer," Journal of Urology, vol. 176, no. 4, pp. 1415-1419, 2006.

[4] A. L. Zietman, M. L. DeSilvio, J. D. Slater et al., "Comparison of conventional-dose vs high-dose conformal radiation therapy in clinically localized adenocarcinoma of the prostate: a randomized controlled trial," Journal of the American Medical Association, vol. 294, no. 10, pp. 1233-1239, 2005.

[5] M. G. Sanda, R. L. Dunn, J. Michalski et al., "Quality of life and satisfaction with outcome among prostate-cancer survivors," New England Journal of Medicine, vol. 358, no. 12, pp. 12501261, 2008.

[6] T. N. Eade, E. M. Horwitz, K. Ruth et al., "A comparison of acute and chronic toxicity for men with low-risk prostate cancer treated with intensity-modulated radiation therapy or $125 \mathrm{i}$ permanent implant," International Journal of Radiation Oncology Biology Physics, vol. 71, no. 2, pp. 338-345, 2008.

[7] J. P. Fine and R. J. Gray, "A Proportional Hazards Model for the Subdistribution of a Competing Risk," Journal of the American Statistical Association, vol. 94, no. 446, pp. 496-509, 1999.

[8] R. J. Gray, "A class of K-sample tests for comparing the cumulative incidence of a competing risk," Annals of Statistics, vol. 16, pp. 1141-1154, 1988.

[9] D. S. Mohan, P. A. Kupelian, and T. R. Willoughby, "Short-course intensity-modulated radiotherapy for localized prostate cancer with daily transabdominal ultrasound localization of the prostate gland," International Journal of Radiation Oncology Biology Physics, vol. 46, no. 3, pp. 575-580, 2000.

[10] P. A. Kupelian, C. A. Reddy, E. A. Klein, and T. R. Willoughby, "Short-course intensity-modulated radiotherapy (70 GY at 2.5 GY per fraction) for localized prostate cancer: preliminary results on late toxicity and quality of life," International Journal of Radiation Oncology Biology Physics, vol. 51, no. 4, pp. 988993, 2001.

[11] D. A. Wilkinson, E. J. Lee, J. P. Ciezki et al., "Dosimetric comparison of pre-planned and or-planned prostate seed brachytherapy," International Journal of Radiation Oncology Biology Physics, vol. 48, no. 4, pp. 1241-1244, 2000.

[12] A. L. Potosky, J. Legler, P. C. Albertsen et al., "Health outcomes after prostatectomy or radiotherapy for prostate cancer: results from the prostate cancer outcomes study," Journal of the National Cancer Institute, vol. 92, no. 19, pp. 1582-1592, 2000.

[13] M. S. Litwin, D. J. Pasta, J. Yu, M. L. Stoddard, and S. C. Flanders, "Urinary function and bother after radical prostatectomy or radiation for prostate cancer: a longitudinal, multivariate quality of life analysis from the cancer of the prostate strategic urologic research endeavor," Journal of Urology, vol. 164, no. 6, pp. 1973-1977, 2000.

[14] J. A. Talcott, J. Manola, J. A. Clark et al., "Time course and predictors of symptoms after primary prostate cancer therapy," Journal of Clinical Oncology, vol. 21, no. 21, pp. 39793986, 2003.

[15] D. M. Latini, J. M. Chan, J. E. Cowan et al., "Health-related quality of life for men with prostate cancer and diabetes: a longitudinal analysis from CaPSURE," Urology, vol. 68, no. 6, pp. 1242-1247, 2006. 
[16] T. E. Schultheiss, W. R. Lee, M. A. Hunt, A. L. Hanlon, R. S. Peter, and G. E. Hanks, "Late GI and GU complications in the treatment of prostate cancer," International Journal of Radiation Oncology Biology Physics, vol. 37, no. 1, pp. 3-11, 1997.

[17] D. M. Herold, A. L. Hanlon, and G. E. Hanks, "Diabetes mellitus: a predictor for late radiation morbidity," International Journal of Radiation Oncology Biology Physics, vol. 43, no. 3, pp. 475-479, 1999.

[18] M. Keyes, S. Miller, V. Moravan et al., "Urinary symptom flare in 712 125I prostate brachytherapy patients: longterm follow-Up," International Journal of Radiation Oncology Biology Physics, vol. 75, no. 3, pp. 649-655, 2009.

[19] M. S.Y. Thong, L. Van De Poll-Franse, R. M. Hoffman et al., "Diabetes mellitus and health-related quality of life in prostate cancer: 5-year results from the Prostate Cancer Outcomes Study," BJU International, vol. 107, no. 8, pp. 1223-1231, 2011.

[20] B. B. Barone, H. C. Yeh, C. F. Snyder et al., "Long-term allcause mortality in cancer patients with preexisting diabetes mellitus: a systematic review and meta-analysis," Journal of the American Medical Association, vol. 300, no. 23, pp. 2754-2764, 2008.

[21] A. V. D'Amico, M. H. Braccioforte, B. J. Moran, and M. H. Chen, "Causes of death in men with prevalent diabetes and newly diagnosed high-versus favorable-risk prostate cancer," International Journal of Radiation Oncology Biology Physics, vol. 77, no. 5, pp. 1329-1337, 2010.

[22] M. P. Sang, K. L. Min, A. S. Soon, and H. Y. Young, "Impact of prediagnosis smoking, alcohol, obesity, and insulin resistance on survival in male cancer patients: National Health Insurance Corporation Study," Journal of Clinical Oncology, vol. 24, no. 31, pp. 5017-5024, 2006.

[23] M. R. Smith, K. Bae, J. A. Efstathiou et al., "Diabetes and mortality in men with locally advanced prostate cancer: RTOG 92-02," Journal of Clinical Oncology, vol. 26, no. 26, pp. 43334339, 2008.

[24] L. V. Van De Poll-Franse, S. Houterman, M. L. G. JanssenHeijnen, M. W. Dercksen, J. W. W. Coebergh, and H. R. Haak, "Less aggressive treatment and worse overall survival in cancer patients with diabetes: a large population based analysis," International Journal of Cancer, vol. 120, no. 9, pp. 1986-1992, 2007.

[25] D. C. Miller, M. G. Sanda, R. L. Dunn et al., "Long-term outcomes among localized prostate cancer survivors: healthrelated quality-of-life changes after radical prostatectomy, external radiation, and brachytherapy," Journal of Clinical Oncology, vol. 23, no. 12, pp. 2772-2780, 2005.

[26] E. J. Hall and A. J. Giaccia, Radiobiology for the Radiologist, Lippincott Williams \& Wilkins, Philadelphia, Pa, USA, 6th edition, 2006.

[27] P. A. Kupelian, V. V. Thakkar, D. Khuntia, C. A. Reddy, E. A. Klein, and A. Mahadevan, "Hypofractionated intensitymodulated radiotherapy (70 Gy at $2.5 \mathrm{~Gy}$ per fraction) for localized prostate cancer: long-term outcomes," International Journal of Radiation Oncology Biology Physics, vol. 63, no. 5, pp. 1463-1468, 2005.

[28] P. A. Kupelian, T. R. Willoughby, C. A. Reddy, E. A. Klein, and A. Mahadevan, "Hypofractionated intensity-modulated radiotherapy (70 Gy at $2.5 \mathrm{~Gy}$ Per Fraction) for localized prostate cancer: Cleveland Clinic Experience," International Journal of Radiation Oncology Biology Physics, vol. 68, no. 5, pp. 1424-1430, 2007.
[29] E. E. Yeoh, R. J. Botten, J. Butters, A. C. Di Matteo, R. H. Holloway, and J. Fowler, "Hypofractionated versus conventionally fractionated radiotherapy for prostate carcinoma: final results of phase III randomized trial," International Journal of Radiation Oncology, Biology, Physics, vol. 81, pp. 1271-1278, 2010. 


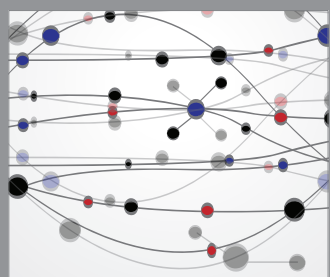

The Scientific World Journal
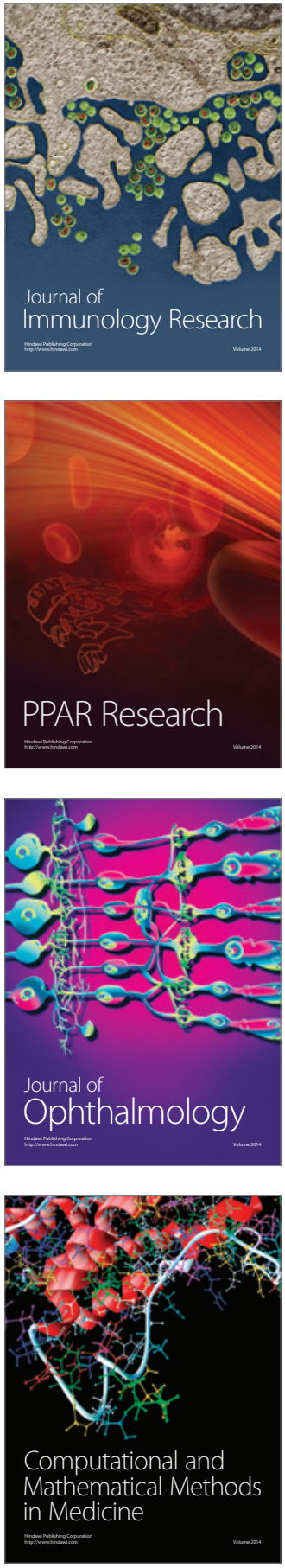

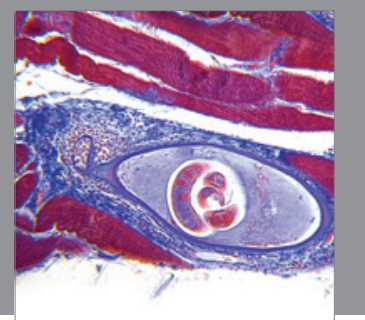

Gastroenterology

Research and Practice
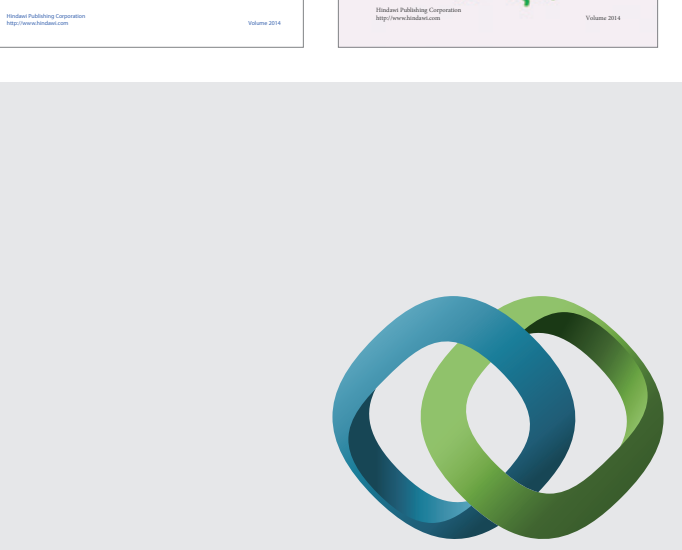

\section{Hindawi}

Submit your manuscripts at

http://www.hindawi.com
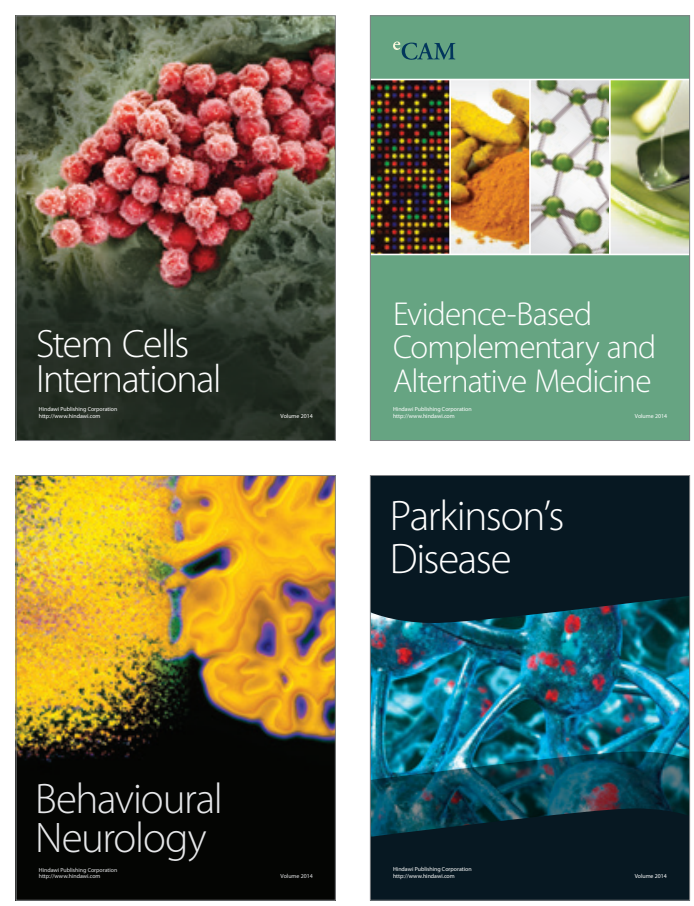

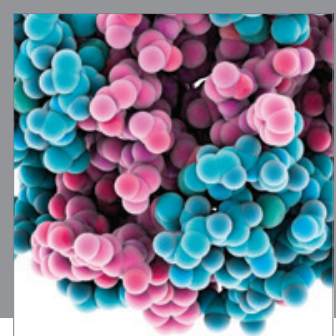

Journal of
Diabetes Research

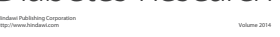

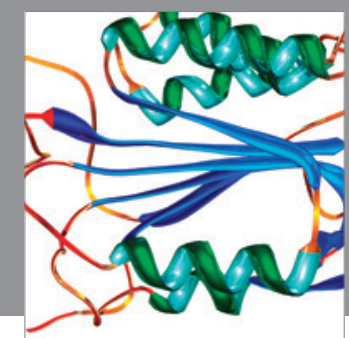

Disease Markers
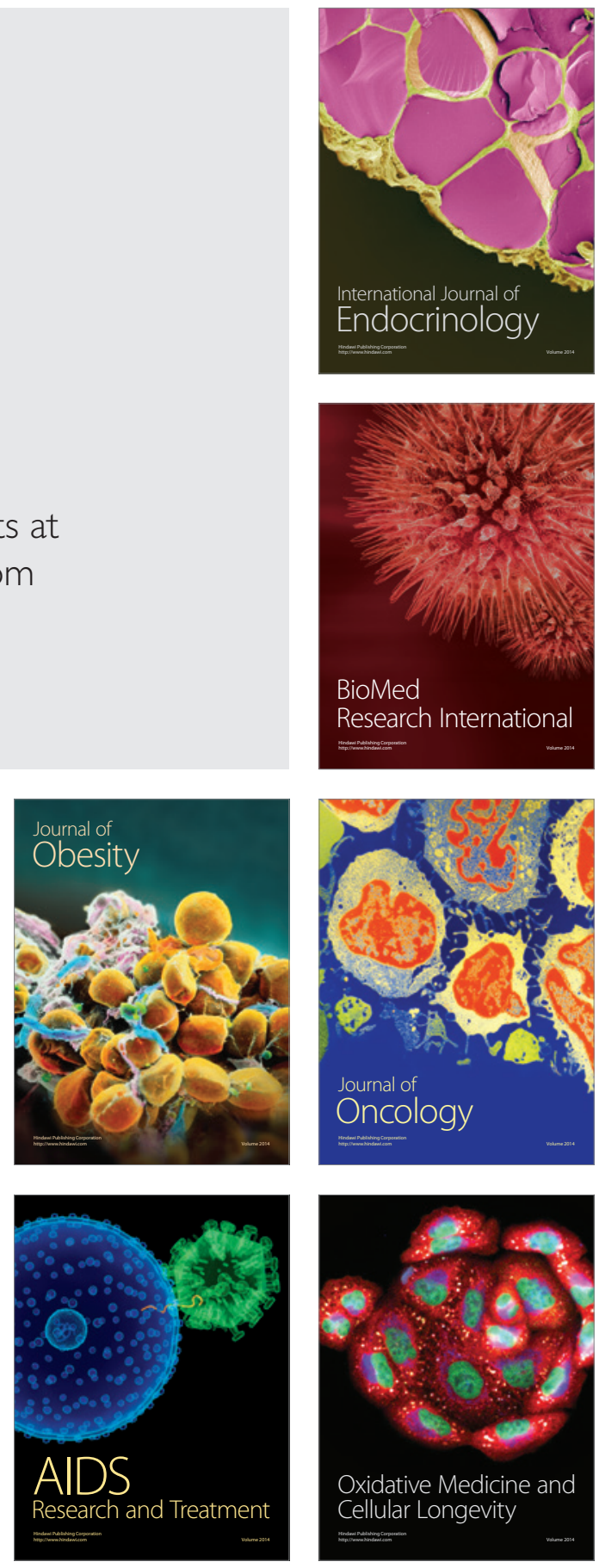